\title{
Impact of Lymphopenia on COVID-19 infection Severity Single-center experience
}

\author{
Elhassadi E*, Morton F, Hourigan A, McDonald L and Elstead C \\ Hematology Department, Laboratory Services, University Hospital Waterford, Waterford - Ireland
}

\begin{abstract}
The World Health Organization (WHO) has declared Coronavirus disease 2019 (COVID-19) as a global public health pandemic. Prudent allocation of resources in a pandemic is essential, and therefore clinical and laboratory biomarkers should be utilized to predict the severity of COVID-19 and allow for resources to be distributed appropriately. A retrospective study was conducted to investigate the association of lymphocyte count with admission rate and disease severity at University Hospital Waterford (UHW) and affiliated sector hospitals. A total of 22,213 samples were received at our Laboratory for COVID-19 testing by PCR. A quarter of the samples (185 cases) were received from COVID-19 Emergency Department pathways at our hospitals. Six per cent of the PCR positive patients were only mildly symptomatic and discharged from ED without blood testing. Overall, 150 (20\%) patients were required hospital admission due to disease severity. Of these, 11 (1.4\%) patients were admitted to ICU. The mortality rate in this cohort was $4.2 \%$ ( 35 patients), and 29 of these were aged over 60 years. The analysis showed that patients with poor outcomes had lower lymphocyte counts. The median lymphocyte counts for ICU and RIP cohorts were $0.81 \mathrm{x} 10^{9} / \mathrm{L}$ (range $0.42-1.66)$ and $0.68 \times 10^{9} / \mathrm{L}(0.21-2.2)$ respectively. Our study showed rates of COVID-19 infection and hospital admissions that are comparable with the reported literature. The impact of lymphopenia on disease severity and mortality is most significant in elderly populations. Therefore, older age and lymphopenia may be used in combination to predict poor prognosis in COVID-19 patients.
\end{abstract}

\section{Introduction}

The World Health Organization (WHO) has declared Coronavirus disease 2019 (COVID-19) as a global public health pandemic. The disease is associated with a high rate of mortality particularly in the elderly population $[1,2]$. While the majority of patients are either asymptomatic or have mild influenza-like illness, some patients will develop severe infection and develop pneumonia, acute respiratory distress syndrome (ARDS), multi-organ failure (MOF), and death may result [3]. Prudent allocation of resources in a pandemic is essential, and therefore clinical and laboratory biomarkers should be utilized to predict the severity of COVID-19 and allow for resources to be distributed appropriately [4]. Lymphopenia, defined as a lymphocyte count less than $1 \times 10^{9} / \mathrm{L}$ (Normal range $1-3 \times 10^{9} / \mathrm{L}$ ), has been associated with severe COVID-19 illness [5-7]; it has also been noted that patients who have died of COVID-19 had significantly lower lymphocyte counts than those who recovered [7]. According to the latest COVID-19 Health Surveillance Monitor Ireland report (03/08/2020), the total confirmed COVID-19 cases since disease out-break is 26,162 , of which 3359 (13\%) required hospitalization. Of these, 437 (1.7\%) patients required Intensive Care Unit (ICU) admission and 1,763 (6.7\%) patients died.

The aim of this study is to evaluate the impact of lymphocyte count on COVID-19 disease severity and patient outcomes in our center.

\section{Objective}

A retrospective study was conducted to investigate the association of lymphocyte count with admission rate and disease severity at University Hospital Waterford (UHW) and affiliated sector hospitals including Wexford General Hospital (WGH), South Tipperary General Hospital (STGH) and St Luke's Hospital Kilkenny (SLH). This study was approved by the Ethics Committee at our center.

\section{Methods}

Data was collected over a three months period (March -June 2020) using the Microbiology Department database; all patients who tested positively for COVID-19 were evaluated. Data collected included age, gender, full blood count, coagulation screen, and C-reactive protein (CRP) as a marker of inflammatory response. Information regarding patient outcomes was also analysed, including length of admission, discharge rate, ICU admission, and mortality rates. A comparison of lymphocyte counts in each group (General medical admission (GM), ICU admission and RIP group) was carried out and these results were validated by using the Mann-Whitney $U$ test to confirm significance.

\section{Results}

A total of 22,213 samples were received at our Laboratory for COVID-19 testing by PCR, including 43 (0.19\%) which were repeat samples and 293 samples (1.3\%) that were rejected/failed. 762 (3.4\%) of samples were reported positive for COVID-19 infection. A quarter of the samples (185 cases) were received from COVID-19 Emergency Department pathways at our hospitals (WGH, STGH, SLH and UHW) $(23 \%, 13.3 \%, 53 \%$ and $10 \%$ respectively). Six per cent of the PCR positive patients were only mildly symptomatic and discharged from ED without blood testing. The median age of the entire COVID-19

${ }^{\star}$ Correspondence to: Ezzat Elhassadi, MD, M.MedSci, FRCPI, FRCPath, Clinical Director for Haematology and Transfusion services, Consultant Haematologist, Honorary Clinical Lecturer, University Hospital Waterford, Dunmore Road, Waterford, X91 ER8E, Ireland, Tel: 051848581; E-mail: ezzat.elhassadi@hse.ie

Key words: coronavirus, COVID-19, lymphocyte count, lymphopenia, elderly

Received: August 25, 2020; Accepted: August 31, 2020; Published: September 03,2020 
positive cohort was 75 years (range $25-99$ ), with a male predominance [M 96 (52\%) vs F 89 (48\%)] (Table 1).

Overall, 150 (20\%) patients were required hospital admission due to disease severity. Of these, $11(1.4 \%)$ patients were admitted to ICU. The majority (82\%) of these patients were subsequently discharged. The mortality rate in this cohort was $4.2 \%$ (35 patients), and 29 of these were aged over 60 years.

Full blood count, coagulation screen, and CRP results were evaluated for all admitted patients $(n=150)$. The analysis showed that patients with poor outcomes had lower lymphocyte counts; there was a clear correlation between lymphopenia and severe COVID-19 infection. The median lymphocyte counts for ICU and RIP cohorts were 0.81 $\mathrm{x} 10^{9} / \mathrm{L}$ (range $0.42-1.66$ ) and $0.68 \times 10^{9} / \mathrm{L}(0.21-2.2)$ respectively. This is in contrast to the GM admission cohort where the median lymphocyte count was normal at $1.09 \times 10^{9} / \mathrm{L}$ (range 0.19-3.9) (Table 2).
The analysis also showed that the association between lymphopenia and composite poor outcome was affected by age. The number of deaths in patients older than 60 years old was 29 patients compared with only 3 deaths in younger patients. The median lymphocyte count in the older population was $0.82 \times 10^{9} / \mathrm{L}$ (range $0.62-3.1$ ), compared with $1.2 \times 10^{9} / \mathrm{L}$ (range 0.25-3.1) in those aged under 60 years. There was no noticeable impact of coagulation screen and CRP results on COVID-19 infection severity. This may be explained by the small number of cases in this study and the fact that these lab parameters were only available in a limited number of patients.

Using the Mann-Whitney U test, mean rank was higher in the GM admission cohort than the ICU/RIP combined cohorts, showing that the lymphocyte count was higher in the GM admission cohort. The Z number was $>1.96$ (3.803) showing there was a statistically significant difference in the lymphocyte count between the GM admission cohort and the ICU/RIP combined cohorts with a p value of $<0.001$ (Figure 1 ). The

Table 1. COVID-19 positive cases and Hospital admission

\begin{tabular}{|c|c|c|}
\hline & Number & $\%$ \\
\hline COVID-19 positive cases & 762 & $3.40 \%$ \\
\hline Hospitals positive cases & 185 & $24 \%$ \\
\hline Hospital Admission & 150 patients & $2 \%$ \\
\hline Median age & 75 years & Range (25-99) \\
\hline Gender distribution & $\mathrm{M}=96$ vs $\mathrm{F}=89$ & 52 vs $48 \%$ \\
\hline WGH & 43 & $23 \%$ \\
\hline STGH & 25 & $13.50 \%$ \\
\hline SLH & 98 & $53 \%$ \\
\hline UHW & 19 & $10 \%$ \\
\hline
\end{tabular}

Table 2. Blood profile of COVID-19 positive cases and the impact of lymphopenia

\begin{tabular}{|l|l|l|l|l|}
\hline Median & All admission & $\mathbf{1 5 0}$ & $\begin{array}{l}\text { GM Admission Cohort } \\
\mathbf{( 1 1 5 )}\end{array}$ & $\begin{array}{l}\text { ICU Cohort } \\
\text { (11) }\end{array}$ \\
\hline WCC & $7.1(2.2-27)$ & $10.3(2.2-27.4)$ & 13.2 & 10.1 \\
\hline HB & $12.5(6.2-18.8)$ & $12.2(8.1-18.8)$ & 9.3 \\
\hline Platelets & $231(76-905)$ & $317(76-905)$ & 306.5 & 11.4 \\
\hline Neutrophils & $5.09(1.56-25)$ & $8.3(1.5-25)$ & 10.1 \\
\hline Lymphocytes Count & $1.01 \times 10^{9}(0.19-3.19)$ & $1.16 \times 10^{9}(0.19-3.19)$ & $0.81 \times 10^{9}(0.42-1.66)$ \\
\hline $1 .<60$-years old & $1.22 \times 10^{9}(0.25-2.4)$ & $1.23 \times 10^{9}(0.25-2.4)$ & $0.78 \times 10^{9}(0.68-1.66)$ \\
\hline $2 .>60$ years old & $0.87 \times 10^{9}(0.19-3.19)$ & $0.96 \times 10^{9}(0.9-3.19)$ & $0.75 \times 10^{9}(0.42-1.29)$ \\
\hline PT & 11.4 & 11.5 & 11.3 & $0.68 \times 10^{9}($ range $0.21-2.2)$ \\
\hline APTT & 29.4 & 29.15 & 26 & $0.82(0.62-3.1)(29$ patients $)$ \\
\hline CRP & 50.1 & 64 & 281 \\
\hline
\end{tabular}

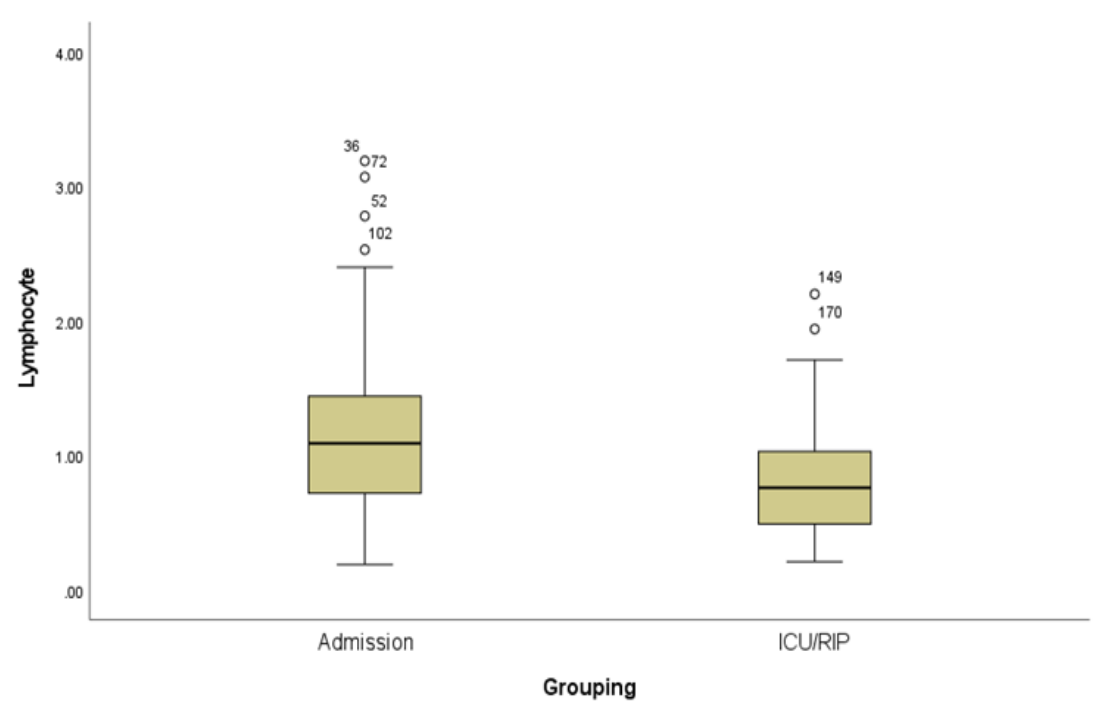

Figure 1. Box plot for lymphocyte count amongst GM admission and ICU/RIP combined cohort showing the distribution and range of lymphocyte count in each group 


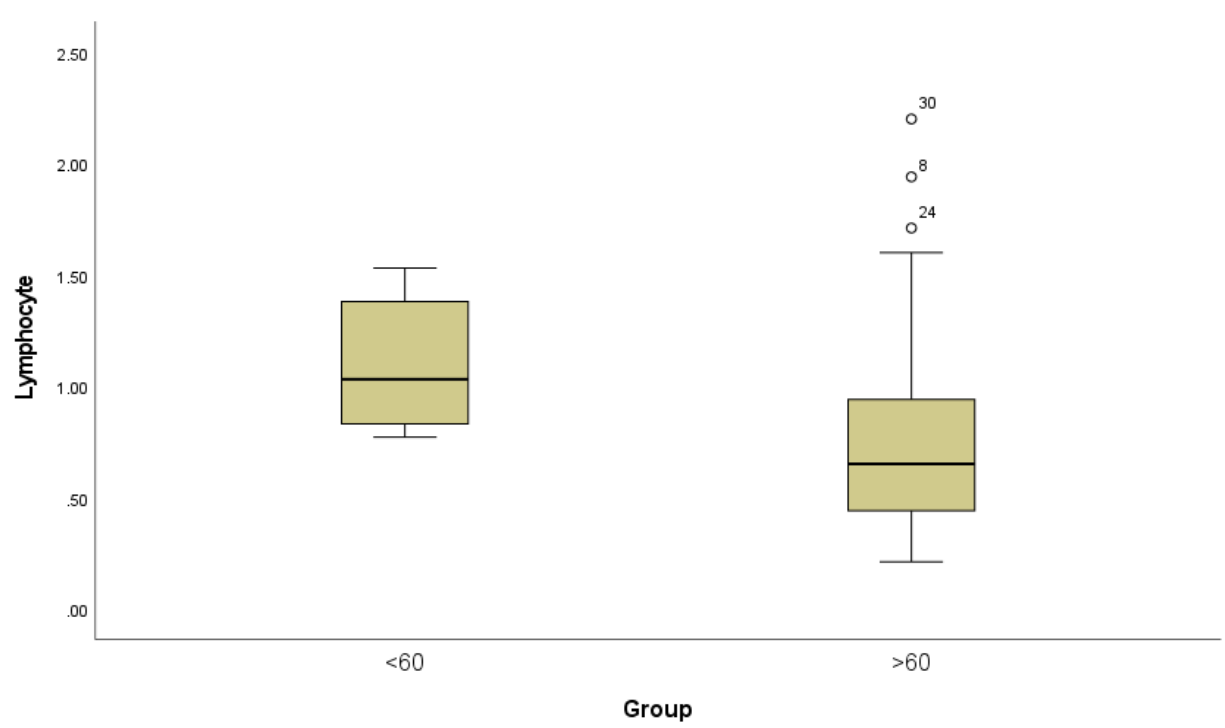

Figure 2. Box plot for lymphocyte count between those $<60$ and $>60$ years of age in the RIP cohort, showing the distribution of the mean and range of lymphocyte count for each group

patients' age (Group1 <60years vs Group2 >60years) and lymphocyte count impact were evaluated and the mean rank is higher in group 1 (patients <60) than group 2 (patients $>60$ ). This showed that the lymphocyte count was higher in patients $<60$ years. The $\mathrm{Z}$ number is $>1.96$ (3.572) showing a statistically significant difference between the lymphocyte count in the two groups $(\mathrm{P}<0.001)$ (Figure 2$)$.

\section{Conclusion}

Our study showed rates of COVID-19 infection and hospital admissions that are comparable with the reported literature. This metaanalysis has shown that lymphopenia on admission was associated with poor outcome in patients with COVID-19. The impact of lymphopenia on disease severity and mortality is most significant in elderly populations. Therefore, older age as clinical parameter and lymphopenia as a laboratory marker may be used in combination to predict poor prognosis in COVID-19 patients.

\section{References}

1. WHO (2020) Coronavirus disease (COVID-19) outbreak, World Health Organization.

2. WHO (2020) Coronavirus disease 2019 (COVID-19) Situation Report-79. World Health Organization.

3. Chen N, Zhou M, Dong X, Qu J, Gong F, et al. (2020) Epidemiological and clinica characteristics of 99 cases of 2019 novel coronavirus pneumonia in Wuhan, China: a descriptive study. Lancet 395: 507-513.

4. Pranata R, Huang I, Lukito A, Raharjo SB (2020) Elevated N-terminal Pro-Brain Natriuretic Peptide is Associated with Increased Mortality in Patients with COVID-19 - Systematic Review and Meta-analysis. Postgrad Med J 2020: 137884. [Crossref]

5. Huang H, Pranata R (2020) Lymphopenia in severe coronavirus disease-2019 (COVID-19): systematic review and meta-analysis. Journal of Intensive Care 8: 36.

6. Yang X, Yu Y, Xu J, Shu H, Xia J, et al. (2020) Clinical course and outcomes of critically ill patients with SARS-CoV-2 pneumonia in Wuhan, China: a single-centered, retrospective, observational study. Lancet Respir Med 2600: 1-7.

7. Ruan Q, Yang K, Wang W, Jiang L, Song J (2020) Clinical predictors of mortality due to COVID-19 based on an analysis of data of 150 patients from Wuhan, China Intensive Care Med 46: 846-848. [Crossref]

Copyright: $\odot 2020$ Elhassadi E. This is an open-access article distributed under the terms of the Creative Commons Attribution License, which permits unrestricted use, distribution, and reproduction in any medium, provided the original author and source are credited. 